\title{
Traitement médical de la maladie De La Peyronie
}

L. WAGNER, P. COSTA

Service d'Urologie-Andrologie C H U de Nimes

\section{RESUME}

La maladie de La Peyronie est caractérisée par la survenue d'une ou plusieurs plaques fibro-inflammatoires au niveau de l'albuginée des corps caverneux. Ce processus est responsable à des degrés divers de douleurs, de courbure en érection ainsi que dans les formes évoluées d'insuffisance érectile.

De très nombreux traitements médicaux ont été proposés pendant la phase active de la maladie. Cependant la comparaison de l'efficacité des différents traitements ne met pas en évidence de différence significative. L'amélioration sur la douleur lors des rapports est observée dans la majorité des cas ; la réduction de la courbure ou de la taille de la plaque chez seulement un tiers des patients. Le traitement idéal sera donc le moins contraignant et le plus dénué d'effets secondaires. Parmi les traitements oraux, la vitaminothérapie $\mathbf{E}$ est celui qui répond le mieux à ces critères. La radiothérapie localisée doit être réservée aux formes très douloureuses résistant aux antalgiques habituels. Une irradiation localisée respectant les doses recommandées ne donne pas de complications.

Parmi les nouveaux traitements médicaux de la maladie de La Peyronie, l'injection locale d'Orgotéine semble donner les meilleurs résultats. Ce traitement permettrait d'obtenir une efficacité plus rapide que celle du traitement par vitamine $E$, en particulier en cas d'association au traitement physique par ionophorèse. Cependant, la confirmation de ces résultats nécessite que soit réalisées des études prospectives randomisées versus placebo.

Mots clés : Courbure de verge, Maladie de La Peyronie, Impuissance.

\section{INTRODUCTION}

Les lésions initiales de la maladie de La Peyronie sont celles d'une vascularite dont l'origine la plus vraisemblable semble auto-immune. Le traitement le plus adapté devrait être étiologique. Cependant des médicaments immunosuppresseurs ne peuvent être administrés de façon prolongée, sans prendre de risques disproportionnés par rapport à la bénignité de l'affection.

Les traitements médicaux visent donc à diminuer ou inhiber les lésions de vascularite, c'est-à-dire l'exsudat plasmatique, la fibrinolyse et l'activation fibro-blastique à l'origine de la chéloïde au niveau de l'albuginée.

Il est donc capital d'identifier le stade de la maladie : phase inflammatoire (active) ou fibreuse (cicatricielle) qui survient en moyenne après 18 mois d'évolution. Seul le premier stade de la maladie justifie un traitement médical.

Parmi les nombreux traitements proposés 
dans la maladie de La Peyronie, certains ont été abandonnés à cause de leur inefficacité ou de leur toxicité. D'autres font l'objet de voies de recherches et sont en cours d'évaluation.

\section{LES TRAITEMENTS MEDICAUX CLASSIQUES}

\section{Procarbazine (Natulan*)}

Médicament cytotoxique, immunosuppresseur dont l' action antifibroblastique est due à une inhibition de la Monoamine Oxydase. Chesney dans une série de 17 patients retrouve plus de $80 \%$ d'amélioration sur la douleur. Les effets secondaires sont de type cytotoxique : troubles digestifs (nausées, vomissements), troubles hématologiques (leucopénie, thrombopénie) et retentissement sur l'épithélium germinal avec possibilité d'azoospermie irréversible [3].

\section{Parabenzoate de Potassium (Potaba*)}

Son action est liée à une augmentation de la disponibilité de l'oxygène intracellulaire, diminuée dans les processus fibrotiques. Il s'agit d'un traitement oral (12 g/jour). Les résultats obtenus sont variables selon les séries avec 16 à $88 \%$ d'amélioration concernant la douleur. Ludwig rapporte une série de 60 patients traités par Potaba pendant 3 mois. Un tiers ont interrompus le traitement (intolérance digestive ou inefficacité). Parmi les 31 patients évalués après traitement l'amélioration de la douleur et de la déformation a été observée dans $1 / 3$ des cas. Les effets secondaires représentés par les hypoglycémies et les troubles digestifs sont fréquents [5].

\section{Les hormones parathyroüdiennes}

Traitement local administré à la dose d'une injection par semaine (50 UI dans la plaque) pendant 8 semaines. Morales rapporte une efficacité modérée avec amélioration des signes objectifs dans environ $1 / 3$ des cas. Les effets secondaires sont représentés par des douleurs importantes à l'injection et un risque potentiel de perturbation du bilan phosphocalcique [7].

\section{Les Corticoüdes}

Traitement local pouvant être délivré par injection dans la plaque à l'aiguille ou sous pression à l'aide du Dermojet* ( 1 injection par mois pendant 6 mois). William dans une série de 42 patients traités pendant 6 mois retrouve une bonne efficacité sur la douleur, améliorée dans $80 \%$ des cas . Cependant une récidive à l'arrêt du traitement a été notée chez un tiers des patients. Les effets secondaires sont essentiellement locaux : douleurs, atrophie ou fibrose sous-cutanée pouvant rendre une prise en charge ultérieure plus complexe [15].

\section{$5^{\circ}$ La Vitaminothérapie E (Tocophérol)}

Traitement oral administré à la dose de 300 $\mathrm{mg} / \mathrm{jour}$. Son action est liée à une activité 'prostaglandine like' entraînant une inhibition de l'activité fibroblastique. Les résultats obtenus sont variables en fonction des séries (9 à $91 \%$ d'amélioration sur la douleur).

Une étude randomisée contre placebo a été réalisée par Pryor chez 60 patients traités pendant 3 mois. Les résultats obtenus ne mettent en évidence de différence significative que concernant l'efficacité sur la douleur lors de l'érection. Aucune différence concernant la réduction de l'incurvation ou de la taille de la plaque n'est retrouvée.

C'est un traitement oral très bien toléré, sans effet secondaire. Son principal inconvénient est d'être un traitement de longue durée (plusieurs mois ou années).

\section{La radiothérapie (irradiation locali- sée)}

Cette irradiation se fait à une dose de 7 à 10 Gy. Il n'existe pas d' efficacité supplémentaire pour des doses plus fortes [12]. Rodrigues dans une série de 38 patients traités par radiothérapie ( 9 Gy délivrés sur 5 jours) retrouve une amélioration de la douleur dans $65 \%$ des cas et de la déformation dans $40 \%$. La douleur est améliorée le plus souvent de façon très rapide (moins de 2 mois). Aucune complication n'est survenue. Les complications rapportées initialement par Burford (1951) et Lowsley (1950) à type d'ulcérations cutanées, de fibrose tissulaire et caverneuse étaient dues à l'utilisation de plaque de Radium et à des doses beaucoup plus élevées. 


\section{LES NOUVEAUX TRAITEMENTS}

Des nouvelles thérapeutiques médicales de la maladie de La Peyronie ont fait l'objet d'évaluation ces dernières années mais la majorité n'ont pas été retenues à cause d'effets secondaires trop importants.

\section{Interféron (alpha 2 b)}

Son action est liée à une inhibition de la prolifération fibroblastique ainsi que de la production de collagène. Wegner sur une série de 25 patients traités par injections locales de 1 million d'UI d'Interféron alpha 2 b par semaine pendant 5 semaines retrouve une efficacité sur la réduction de taille de la plaque (diminution importante dans $30 \%$ des cas). Cependant les autres symptômes n'ont pas étés évalués. Des effets secondaires à type de fièvre et myalgies sont survenus chez 4 patients avec nécessité d'arrêt du traitement chez 2 d'entre eux [14].

\section{La collagènase}

Enzyme bactérienne purifiée (Clostridium) qui attaque spécifiquement le collagène. Gelbard dans une étude randomisée versus placebo réalisée chez 49 patients (injection unique) retrouve une différence significative concernant l'amélioration de la courbure et la réduction de la taille de la plaque. L'efficacité sur la douleur en érection n'est pas évaluée .Les effets secondaires rapportés sont limités à la survenue d'ecchymoses transitoires chez 3 patients [2].

\section{La Colchicine}

Son action est liée à une inhibition de l'activité xanthine oxydase et donc de la production de radicaux libres responsable du processus de fibrose. Akkus dans une série de 24 patients traités pendant 3 à 5 mois retrouve une amélioration de la douleur dans $78 \%$ des cas, de la courbure dans $37 \%$ et une réduction de taille de la plaque dans $50 \%$. Malheureusement, des effets secondaires gastro-intestinaux importants ont conduit à l'arrêt du traitement chez 4 patients[1].

\section{Le Tamoxifène}

Son action est liée à une stimulation de la synthèse de TGF Bêta par les fibroblastes entraî- nant une désactivation des macrophages et une action suppressive sur les lymphocytes $\mathrm{T}$.

Ralph et Pryor dans une série de 36 patients traités par $40 \mathrm{mg} /$ jour en 2 prises orales pendant 3 mois retrouvent une amélioration concernant la douleur dans $80 \%$ des cas, une réduction de la courbure dans $35 \%$ et de la taille de la plaque dans $34 \%$ des cas. Des effets secondaires sont survenus chez 5 patients : troubles de la libido chez 3 d'entre eux et bouffées de chaleur chez 2 autres [10].

\section{Le Vérapamil (Isoptine)}

Inhibiteur calcique dont l'action serait lié à une stimulation de l'activité collagènase et une diminution de la synthèse de collagène.

Levine rapporte en 1994 une série de 12 patients traités par 2 injections par semaine dans la plaque pendant 6 mois puis en 1995 une série de 18 patients traités par une seule injection toutes les 2 semaines pendant 6 mois. Les résultats sont semblables avec diminution de la douleur dans $90 \%$ des cas, de la courbure dans $42 \%$ et une réduction de taille de la plaque dans $30 \%$ des cas. Les effets secondaires sont représentés par des douleurs à l'injection et les risques potentiels d'arythmie cardiaque [4].

Deux études récentes remettent en question l'efficacité du verapamil . La première étude non randomisée contre placebo (7 patients dans chaque groupe) ne retrouve pas de différence significative concernant l'amélioration de la courbure [11]. La seconde étude randomisée versus corticoïdes et placebo chez 29 patients ne retrouve pas de différence significative entre les groupes concernant la réduction de la taille de la plaque [13].

\section{L'Orgotéine :}

Métalloprotéine anti-inflammatoire possédant une activité superoxyde desmutase entrâ̂nant une diminution des radicaux libres extra-cellulaires et donc une activité antiinflammatoire et anti-fibroblastique.

Primus dans une étude interessant 18 patients traités pendant 10 mois ( 1 injection par mois) retrouve une amélioration de la douleur dans $100 \%$ des cas et de la courbure dans 
$38 \%$. Les effets secondaires sont limités à une hypotension artérielle chez 1 patient (hypersensibilité au produit) [8].

Montorsi dans une série de 40 patients traités par Orgotéine couplé à une ionophorèse retrouve une efficacité.sur la douleur dans 100 $\%$ des cas et sur la courbure dans $62 \%$. La tolérance du traitement semble excellente. La ionophorèse correspond à l'administration percutanée d'un courant électrique de faible intensité (4 mA). Elle améliorerait la diffusion du produit injecté à travers la plaque.

\section{CONCLUSION}

Il ne semble pas exister de différence significative concernant les résultats des multiples traitements proposés. En moyenne, l'amélioration sur la douleur lors des rapports est observée dans $80 \%$ des cas . La réduction de la courbure ou de la taille de la plaque est observée en moyenne dans un tiers des cas .

Le traitement idéal sera donc le moins contraignant et le plus dénué d'effets secondaires. Parmi les traitements oraux, la vitaminothérapie $E$ est celui qui répond le mieux à ces critères. Il s'agit cependant d'un traitement de longue durée dont l'efficacité nécessite une prise continue pendant plusieurs mois ou années. L'irradiation localisée, à condition de respecter les doses recommandées ne donne pas de complications et reste le traitement le plus rapidement efficace sur les douleurs. Le traitement par injections locales d'Orgotéine semble donner de bons résultats surtout quand il est associé au traitement physique par Ionophorèse. Il possède très peu d'effets secondaires et une efficacité qui paraît plus rapide que les traitements type vitaminothérapie $\mathrm{E}$.

L'amélioration de la prise en charge médicale de la maladie de La Peyronie passera par une meilleure connaissance de la physiopathologie de la maladie, ce qui nécessite la réalisation d'études histologiques avec biopsie de la plaque, mais aussi par une meilleure évaluation de l'efficacité des traitements médicaux imposant la réalisation d'études prospectives randomisées versus placebo .

\section{REFERENCES}

1. AKKUS E., CARRIER S., REHMAN J., BREZA J. KADIOGLU A. LUE T.F. Is Colchicine effective in Peyronie's disease ? Urology , (1994) , 44,2 : 291-295.

2. GELBARD M.K., JAMES K., RIACH P., DOREY F. Collagenase versus placebo in the treatment of Peyronie's disease : a double-blind study. J Urol , (1993), 149, $1: 56-58$.

3. CHESNEY J. Peyronie's Disease . Br J Urol (1975), $47: 209-218$.

4. LEVINE L.A., MERRICK P.F., LEE R.C. Intralesional Verapamil injection for the treatment of Peyronie's disease . J Urol (1994) ,151, 6 : 1522-1524.

5. LUDWIG G. Evaluation of Conservative Therapeutic Approaches to Peyronie's Disease. Urol Int (1991) ; 47 : 236-239.

6. MONTORSI F., GUAZZONI G., BOCCIARDI A. et Coll. Transdermal electro motive multi-drug administration, for Peyronie's disease : a randomised, double blind , placebo-controlled. J Urol (1995), 153 : 472A (\# 974).

7. MORALES A., BRUCE A.W. The treatment of Peyronie's disease with parathyroid hormone. J Urol (1975) , 114, $6: 901-902$.

8. PRIMUS G. Orgotein in Treatment of Plastic induration of the penis . Int Urol Nephrol, (1993), 25, 2 : 169-172.

9. PRYOR $\mathrm{d}$. Controlled clinical trial of vitamin $\mathrm{E}$ in Peyronie's disease . Progress in Reproductive Biology and Medecine. Vol 9, 1983, p41-45.

10. RALPH D.J., BROOKS M.D., BOTTAZZO G. F., PRYOR J.P. The treatment of Peyronie's disease with tamoxifen . Br J Urol (1992) , 70, 6 : 648-651.

11. REHMAN J., BENET A., MELMAN A. Use of intralesional Verapamil to dissolve Peyronie's disease plaque : a long terme single blind study. J Urol, 1996, $155,5,633 \mathrm{~A}$ (\#1289).

12. RODRIGUES C.I., NJO K.H., KARIM A.B. Results of radiotherapy and vitamine $\mathrm{E}$ in the treatment of Peyronie's disease . Int J Radiat Oncol , (1995), 31, 3 : 571-576.

13. TELÖKEN C., VACCARO F., DA ROS C. et Coll . Objective evaluation of non-surgical approach for Peyronie's disease . J Urol , 1996, 155,5, 633A (\#1290) .

14.WEGNER H.E., ANDRESEN R., KNIPSEL H.H., MILLER K. Treatment of Peyronie's disease with local interferon-alpha $2 \mathrm{~b}$. European Urology (1995), $28,3: 236-240$.

15. WILLIAMS G., GREEN N.A . The Non-surgical Treatment of Peyronie's Disease . Br J Urol (1980), $52: 392-395$. 


\section{ABSTRACT \\ Medical treatment of La Peyronie's disease}

L. WAgner, P.Costa

Peyronie's disease is characterized by the presence of a scar, called a plaque, involving the tunica albuginea of the corpora carvernosa. This may be associated early in its course with painful erections and subsequently with deformity of the erect penis.

During the acute phase, multiple conservative treatments have been aimed at control of clinical symptoms.

Comparative studies between these therapies show no difference in terms of results. In addition, no controlled study has been able to point out that the patients receiving these treatments do better than those receiving no treatment.

We usually give vitamin $E$ because this oral therapy is easy to use and have no side effects. Persistence of pain is, to our minds, the only indication for radiation therapy. A small dose of radiation delivered by a soft modality can allay residual pain.

New conservative treatments of Peyronie's disease have been reported. Orgotein injections give the most encouraging results, especially when associated with transdermal electromotive administration. To confirme these results we require prospective double-blind studies.

Key Words : Male, Penile induration, Genital diseases, Impotence, Peyronie's disease. 